Journal of Mathematics and Statistics 2 (1): 339-342, 2006

ISSN 1549-3644

(C) 2006 Science Publications

\title{
On Some Stability Results for Fixed Point Iteration Procedure
}

\author{
M. O. Olatinwo, O. O. Owojori and C. O. Imoru \\ Department of Mathematics, Obafemi Awolowo University, Ile-Ife, Nigeria.
}

\begin{abstract}
In this study, we establish that both the Mann and Ishikawa iteration processes are T-stable for the mappings $\mathrm{T}$ satisfying a more general contractive definition than that of Osilike ${ }^{[1]}$. The results obtained generalize some of the recent results of Osilike ${ }^{[1]}$ which are themselves generalizations and extensions of some of the results of Harder and Hicks ${ }^{[2]}$ and Rhoades ${ }^{[3,4]}$.
\end{abstract}

Key words: Stability results, fixed point iteration procedure

\section{INTRODUCTION}

Let $(\mathrm{E}, \mathrm{d})$ be a complete metric space and $\mathrm{T}: \mathrm{E} \rightarrow$ E a selfmap of $E$ and $F(T)=\left\{p \in E: T_{p}=p\right\}$, the set of fixed points of $\mathrm{T}$. For $\mathrm{x}_{\mathrm{o}} \in \mathrm{E}$, define sequence $\left\{\mathrm{x}_{\mathrm{x}}\right\}_{n=o}^{\infty}$ iteratively by

$\mathrm{x}_{\mathrm{n}+1}=\mathrm{f}\left(\mathrm{T}, \mathrm{x}_{\mathrm{n}}\right), \mathrm{n}=0,1,2, \ldots$

Suppose $\left\{\mathrm{X}_{n}\right\}_{n=o}^{\infty}$ converges to a fixed point $\mathrm{p}$ of $\mathrm{T}$ and let $\varepsilon_{\mathrm{n}}=\mathrm{d}\left(\mathrm{y}_{\mathrm{n}+1}, \mathrm{f}\left(\mathrm{T}, \mathrm{y}_{\mathrm{n}}\right)\right)$, where $\left\{\mathrm{y}_{\mathrm{n}}\right\}_{n=o}^{\infty} \subset \mathrm{E}$. Then, the iteration procedure (I) is said to be T-stable or stable with respect to $\mathrm{T}$ if and only if $\lim _{n \rightarrow \infty} \mathcal{E}_{n}=0$ implies $\lim _{n \rightarrow \infty} y_{n}=p$.

Harder and Hicks ${ }^{[2]}$ established several stability results under various contractive conditions using the above concept. Rhoades ${ }^{[3,4]}$ extended the results of Harder and Hicks $^{[2]}$ to other classes of contractive mappings. In Rhoades ${ }^{[4]}$, the following contractive definition was considered : there exists a constant $\mathrm{c} \in$ $[0,1)$, such that for each $\mathrm{x}, \mathrm{y} \in \mathrm{E}$,

$\mathrm{d}(\mathrm{Tx}, \mathrm{Ty}) \leq \mathrm{c} \max \left\{\mathrm{d}(\mathrm{x}, \mathrm{y}), \frac{1}{2}[\mathrm{~d}(\mathrm{x}, \mathrm{Tx})+\mathrm{d}(\mathrm{y}, \mathrm{Ty})]\right.$,

$\mathrm{d}(\mathrm{x}, \mathrm{Ty})], \mathrm{d}(\mathrm{x}, \mathrm{Ty}), \mathrm{d}(\mathrm{y}, \mathrm{Tx})\}$

Using (2), Rhoades ${ }^{[4]}$ established several stability results which are generalizations and extensions of most of the results of Harder and Hicks ${ }^{[2]}$ and Rhoades $^{[5]}$. It was shown in Rhoades ${ }^{[6]}$ that if $T$ satisfies (2) then,

$\mathrm{d}(\mathrm{Tx}, \mathrm{Ty}) \leq \frac{c}{1-c} \mathrm{~d}(\mathrm{x}, \mathrm{Ty})+\mathrm{c} \mathrm{d}(\mathrm{x}, \mathrm{y})$

Osilike $^{[1]}$ employed the following contractive definition: for each $\mathrm{x}, \mathrm{y} \in \mathrm{E}$, there exist constants $\mathrm{a} \in$ $[0,1)$ and $L \geq 0$ such that

$d(T x, T y) \leq \operatorname{Ld}(x, T x)+a d(x, y)$.
Using (3), Osilike ${ }^{[1]}$ proved several stability results which are generalizations and extensions of most of the results of Rhoades ${ }^{[4]}$.

Employing the same contractive definitions as in Harder and Hicks ${ }^{[2]}$, Berinde ${ }^{[7]}$ proved the same stability results for the same iteration procedures by an alternative method.

In this study, we extend some of the recent results of Berinde $^{[7]}$, Osilike ${ }^{[1]}$ and Rhoades ${ }^{[4]}$ to a more general contractive definition.

Preliminaries: In the sequel, we shall employ the following contractive definition. For each $\mathrm{x}, \mathrm{y} \in \mathrm{E}$, there exist a constant $b \in[0,1\}$, and a continuous, monotone increasing function $\varphi: \Re_{+} \rightarrow \mathfrak{R}_{+}$with $\varphi(0)$ $=0$, such that

$\mathrm{d}(\mathrm{Tx}, \mathrm{Ty}) \leq \varphi(\mathrm{d}(\mathrm{x}, \mathrm{Tx}))+\mathrm{bd}(\mathrm{x}, \mathrm{y})$.

The contractive definition (4) is more general than those considered by Berinde ${ }^{[7]}$, Harder and Hicks ${ }^{[2]}$, Rhoades $^{[3,4]}$ and Osilike ${ }^{[1]}$. This is evident by specifying $\varphi$ in (4) as follows. If $\varphi(u)=\mathrm{Lu}$ in (4) above, where $\mathrm{L} \geq 0$ is a constant, then we obtain the contractive mapping of Osilike ${ }^{[1]}$ which is itself a generalization of those in Harder and $\mathrm{Hicks}^{[2]}$, Berinde $^{[7]}$ and Rhoades ${ }^{[4]}$. Also, if $\mathrm{L}=\mathrm{mb}$, where $\mathrm{m}=$ $(1-b)^{-1}, b \in[0,1)$, we obtain the contractive mapping considered by Rhoades ${ }^{[4]}$.

Also, if $\mathrm{L}=2 \delta, \quad \mathrm{b}=\delta$, where $\delta=\max$ $\left\{\alpha \frac{\beta}{1-\beta}, \frac{\gamma}{1-\gamma}\right\}, 0 \leq \alpha<1$

$0 \leq \beta<0.5, \quad 0 \leq \Upsilon \leq 0.5$, then we obtain the Zamfirescu's contractive definition which was employed in Harder and Hicks ${ }^{[2]}$ and Berinde ${ }^{[7]}$. Furthermore, if $\varphi(u)=0$, then (4) reduces to $d(T x, T y)$ $\leq \mathrm{bd}(\mathrm{x}, \mathrm{y}), \quad \mathrm{b} \in[0,1)$ which is another contractive definition used by Harder and Hicks ${ }^{[2]}$ and Berinde ${ }^{[7]}$.

In the sequel, we shall establish stability results for the following iteration procedures: 
i. The Mann Iteration Process ${ }^{[1,6]}$, which is defined for arbitrary $\mathrm{x}_{\mathrm{o}} \in \mathrm{E}$ by $\mathrm{x}_{\mathrm{n}+1}=f\left(\mathrm{~T}, \mathrm{x}_{\mathrm{n}}\right)=\left(1-\alpha_{\mathrm{n}}\right) \mathrm{x}_{\mathrm{n}}$ $+\alpha_{\mathrm{n}} \mathrm{Tx}_{\mathrm{n}}, \quad \mathrm{n} \geq 0$, Where, $\left\{\alpha_{\mathrm{n}}\right\}_{n=o}^{\infty}$ is a real sequence satisfying $\alpha_{0}=1,0 \leq \alpha_{n} \leq 1$, for $n>0$ and $\sum_{n=o}^{\infty} \alpha_{n}=\infty$.

ii. The Ishikawa Iteration Process ${ }^{[1,6]}$ which is defined for arbitrary $\mathrm{x}_{\mathrm{o}} \in \mathrm{E}$ by:

$$
\left.\begin{array}{l}
Z_{n}=\left(1-\beta_{n}\right) x_{n}+\alpha_{n} T x_{n} \\
x_{n+1}=f\left(T, X_{n}\right)=\left(1-\alpha_{n}\right) x_{n}+\alpha_{n} T x_{n},
\end{array}\right\}
$$

Where, $\left\{\alpha_{n}\right\}_{n=o}^{\infty}$ and $\left\{\beta_{n}\right\}_{n=o}^{\infty}$ are real sequences satisfying $0 \leq \alpha_{n} \leq \beta_{n} \leq 1$ for all $\mathrm{n} \geq 0$, and $\lim _{n \rightarrow \infty} \beta_{n}=0$ and $\sum_{n=o}^{\infty} \alpha_{n} \beta_{n}=\infty$. We shall employ the following lemmas in the proofs of the stability results.

Lemma 1: Let (E, d) be a complete metric space, and $\mathrm{T}: \mathrm{E} \rightarrow \mathrm{E}-$ a selfmap of E satisfying (4). Let $\mathrm{x}_{0} \in \mathrm{E}$ and $x_{n+1}=T x_{n}, n \geq 0$. Suppose $T$ has a fixed point $p$ and $\varphi: \Re_{+} \rightarrow \Re_{+}=[0, \infty)$ is a continuous monotone increasing function such that $\varphi(0)=0$. Then, $\lim _{n=\infty} \varphi\left(d\left(x_{n}, \mathrm{Tx}_{\mathrm{n}}\right)\right)=0$.

Proof: From (4) and the hypothesis of the Lemma, we have:

$$
\begin{aligned}
\mathrm{d}\left(\mathrm{x}_{\mathrm{n}+1}, \mathrm{p}\right) & =\mathrm{d}\left(\mathrm{Tx}_{\mathrm{n}}, \mathrm{Tp}\right)=\mathrm{d}\left(\mathrm{Tp}, \mathrm{Tx}_{\mathrm{n}}\right) \\
& \leq \varphi(\mathrm{d}(\mathrm{p}, \mathrm{Tp}))+\mathrm{bd}\left(\mathrm{p}, \mathrm{x}_{\mathrm{n}}\right) \\
& =\mathrm{bd}\left(\mathrm{x}_{\mathrm{n}}, \mathrm{p}\right) \leq \mathrm{b}^{2} \mathrm{~d}\left(\mathrm{x}_{\mathrm{n}-1}, \mathrm{p}\right) \leq \ldots \\
& \leq \mathrm{b}^{\mathrm{n}+1} \mathrm{~d}\left(\mathrm{x}_{\mathrm{o}}, \mathrm{p}\right) \rightarrow 0, \quad \text { as } \mathrm{n} \rightarrow \infty
\end{aligned}
$$

By triangle inequality and (4), we have:

$$
\begin{aligned}
\mathrm{d}\left(\mathrm{x}_{\mathrm{n}}, T \mathrm{~T}_{\mathrm{n}}\right) & \leq \mathrm{d}\left(\mathrm{x}_{\mathrm{n}}, \mathrm{p}\right)+\mathrm{d}\left(\mathrm{p}, \mathrm{Tx}_{\mathrm{n}}\right) \\
& =\mathrm{d}\left(\mathrm{x}_{\mathrm{n}}, \mathrm{p}\right)+\mathrm{d}\left(\mathrm{Tp}, T \mathrm{x}_{\mathrm{n}}\right) \\
& \leq \mathrm{d}\left(\mathrm{x}_{\mathrm{n}}, \mathrm{p}\right)+\varphi(\mathrm{d}(\mathrm{p}, T \mathrm{p}))+\mathrm{bd}\left(\mathrm{p}, \mathrm{x}_{\mathrm{n}}\right) \\
& =(1+\mathrm{b}) \mathrm{d}\left(\mathrm{x}_{\mathrm{n}}, \mathrm{p}\right) \rightarrow 0 \text { as } \mathrm{n} \rightarrow \infty
\end{aligned}
$$

Thus, $\lim _{n=\infty} \mathrm{d}\left(\mathrm{x}_{\mathrm{n}}, \mathrm{Tx}_{\mathrm{n}}\right)=0$.

But $\varphi$ is continuous, therefore we have :

$\lim _{n \rightarrow \infty} \varphi\left(d\left(x_{n}, T x_{n}\right)=\varphi\left(\lim _{n \rightarrow \infty} d\left(x_{n}, T x_{n}\right)\right)=0\right.$,

This completes the proof of the Lemma.

Remark 1: The operator $\mathrm{T}$ in Lemma 1 is not necessarily a Picard operator.

Lemma 2: Let $(\mathrm{E},\|\|$.$) be a normed linear space, and$ let $\mathrm{T}: \mathrm{E} \rightarrow \mathrm{E}$ be a selfmap of E satisfying (4). Suppose $\mathrm{T}$ has a fixed point $\mathrm{p}$. Let $\left\{\mathrm{x}_{\mathrm{n}}\right\}_{n=o}^{\infty}$ be the Ishikawa iteration process with $\left\{\alpha_{n}\right\}_{n=o}^{\infty}$ and $\left\{\beta_{\mathrm{n}}\right\}_{n=o}^{\infty}$ satisfying

i. $\quad \alpha_{0}=1$;

ii. $0 \leq \alpha_{n}, \beta_{n} \leq 1, \quad n \geq 0$; iii. $\sum_{n=o}^{\infty} \alpha_{j}=\infty$;

iv. $\sum_{n=o}^{\infty} \prod_{k=j+1}^{n}\left(1-\alpha_{k}+b \alpha_{k}\right)$ converges.

Suppose $\varphi: \mathfrak{R}_{+} \rightarrow \mathfrak{R}_{+}$is a continuous monotone increasing function such that

$\varphi(0)=0$. Let $\left\{\mathrm{y}_{\mathrm{n}}\right\}_{n=o}^{\infty} \subset \mathrm{E}$ and define.

$s_{\mathrm{n}}=\left(1-\beta_{\mathrm{n}}\right) \mathrm{y}_{\mathrm{n}}+\beta_{\mathrm{n}} \mathrm{Ty}_{\mathrm{n}}, \mathrm{n} \geq 0$

$\varepsilon_{\mathrm{n}}=\left\|\mathrm{y}_{\mathrm{n}+1}-\left(1-\alpha_{\mathrm{n}}\right) \mathrm{y}_{\mathrm{n}}-\alpha_{\mathrm{n}} \mathrm{Ts}_{\mathrm{n}}\right\|$

Then,

$$
\begin{aligned}
\| \mathrm{y}_{\mathrm{n}+1}-\mathrm{p} & \|\leq\| \mathrm{x}_{\mathrm{n}+1}-\mathrm{p} \|+\sum_{j=o}^{\infty} \Pi_{k=j+1}^{n} \\
& \left.\left(1-\alpha_{\mathrm{k}}+\mathrm{b} \alpha_{\mathrm{k}}\right) \varphi\left\|\mathrm{z}_{\mathrm{j}}-\mathrm{T} \mathrm{z}_{\mathrm{j}}\right\|\right) \\
& +\sum_{j=o}^{\infty} \alpha_{\mathrm{j}} \beta_{\mathrm{j}} \Pi_{k=j+1}^{n}\left(1-\alpha_{\mathrm{k}}+\mathrm{b} \alpha_{\mathrm{k}}\right) \\
& \varphi\left(\left\|\mathrm{x}_{\mathrm{j}}-\mathrm{T} \mathrm{x}_{\mathrm{j}}\right\|\right) \\
& +\Pi_{k=o}^{n}\left(1-\alpha_{\mathrm{k}}+\mathrm{b} \alpha_{\mathrm{k}}\right)\left\|\mathrm{x}_{\mathrm{o}}-\mathrm{y}_{\mathrm{o}}\right\| \\
& +\sum_{j=o}^{\infty} \Pi_{k=j+1}^{n}\left(1-\alpha_{\mathrm{k}}+\mathrm{b} \alpha_{\mathrm{k}}\right) \varepsilon_{\mathrm{j}},
\end{aligned}
$$

Where the product is 1 when $\mathrm{j}=\mathrm{n}$.

Proof: Using (4) and the triangle inequality, we have the following:

$$
\begin{aligned}
\left\|y_{n+1}-p\right\| & \leq\left\|y_{n+1}-x_{n+1}\right\|+\left\|x_{n+1}-p\right\| \\
& \leq\left\|y_{n+1}-p\right\|+\left\|y_{n+1}-\left(1-\alpha_{n}\right) y_{n}-\alpha_{n} T s_{n}\right\| \\
& +\|\left(1-\alpha_{n} y_{n}-\alpha_{n} T s_{n}-x_{n+1} \|\right. \\
& =\left\|x_{n+1}-p\right\|+\varepsilon_{j}+\| \\
& \left(1-\alpha_{n}\right) y_{n}-\alpha_{n} T s_{n}-\left(1-\alpha_{n}\right) x_{n}-\alpha_{n} T z_{n} \| \\
& \leq\left\|x_{n+1}-p\right\|+\left(1-\alpha_{n}\right)+\left\|x_{n}-y_{n}\right\| \\
& +\alpha_{n}\left\|T z_{n}-T s_{n}\right\|+\varepsilon_{n} \\
& \leq\left\|x_{n+1}-p\right\|+\left(1-\alpha_{n}\right)\left\|x_{n}-y_{n}\right\| \\
& +\alpha_{n}\left[\varphi\left(\left\|z_{n}-T z_{n}\right\|\right)+b\left\|z_{n}-s_{n}\right\|\right]+\varepsilon_{n} \\
& =\left\|x_{n+1}-p\right\|+\left(1-\alpha_{n}\right)\left\|x_{n}-y_{n}\right\| \\
& +\alpha_{n} \varphi\left(\left\|z_{n}-T z_{n}\right\|\right)+\alpha_{n} b\left\|z_{n}-s_{n}\right\|+\varepsilon_{n}
\end{aligned}
$$

Observe that

$$
\begin{aligned}
\left\|z_{n}-s_{n}\right\| & =\left\|\left(1-\beta_{n}\right) x_{n}+\beta_{n} T x_{n}-\left(1-\beta_{n}\right) y_{n}-\beta_{n} T y_{n}\right\| \\
& \leq\left(1-\beta_{n}\right) \|\left(x_{n}-y_{n}\left\|+\beta_{n}\right\| T x_{n}-T y_{n} \|\right. \\
& \leq\left(1-\beta_{n}\right)\left\|x_{n}-y_{n}\right\|+\beta_{n}\left(\varphi\left\|x_{n}-T x_{n}\right\|\right) \\
& \left.+b\left\|x_{n}-y_{n}\right\|\right) \\
& =\beta_{n} \varphi\left(\left\|x_{n}-T x_{n}\right\|\right)+\left(1-\beta_{n}+b \beta_{n}\right)\left\|x_{n}-y_{n}\right\| \\
& \leq \beta_{n} \varphi\left(\left\|x_{n}-T x_{n}\right\|\right)+\left\|x_{n}-y_{n}\right\| .
\end{aligned}
$$

Substituting (7) into (6), we have

$\left\|y_{n+1}-p\right\|_{\leq} \leq y_{n+1}-p \|+\alpha_{n} \varphi$

$\left(\left\|z_{n}-T z_{n}\right\|\right)+b \alpha_{n} \beta_{n} \varphi\left(\left\|x_{n}-T x_{n}\right\|\right)$

$+\left(1-\alpha_{n}+b \alpha_{n}\right)\left\|x_{n}-y_{n}\right\|+\varepsilon_{n}$

Moreover,

$$
\begin{aligned}
\left\|y_{n}-x_{n}\right\| & \leq\left\|y_{n}-\left(1-\alpha_{n-1}\right)_{y n-1}-\alpha_{n-1} T s_{n}\right\| \\
& +\left\|\left(1-\alpha_{n-1}\right) y_{n-1}+\alpha_{n-1} T_{n-1}-x_{n}\right\| \\
& =\varepsilon_{n-1}+\|\left(1-\alpha_{n-1}\right) y_{n-1}-\alpha_{n-1} T s_{n-1} \\
& -\left(1-\alpha_{n-1}\right) x_{n-1}-\alpha_{n-1} T z_{n-1} \|
\end{aligned}
$$




$$
\begin{aligned}
& \leq\left(1-\alpha_{n-1}\right)\left\|x_{n-1}-y_{n-1}\right\|+\alpha_{n-1} \| \\
& T z_{n-1}-T s_{n-1} \|+\varepsilon_{n-1} \\
& \leq\left(1-\alpha_{n-1}\right)\left\|x_{n-1}-y_{n-1}\right\|+\alpha_{n-1} \\
& \left(\varphi\left(\left\|z_{n-1}-T z_{n-1}\right\|\right)\right. \\
& \left.+b\left\|z_{n-1}-s_{n-1}\right\|\right)+\varepsilon_{n-1} \\
& =\alpha_{n-1} \varphi\left(\left\|z_{n-1}-T z_{n-1}\right\|\right)+\left(1-\alpha_{n-1}\right)\left\|x_{n-1}-y_{n-1}\right\| \\
& +b \alpha_{n-1}\left\|z_{n-1}-s_{n-1}\right\|+\varepsilon_{n-1}
\end{aligned}
$$

Similarly, from (7), we have:

$\left.\left\|z_{n-1}-s_{n-1}\right\|\right) \leq \beta_{n-1} \varphi\left(\left\|x_{n-1}-T x_{n-1}\right\|\right)$

$+\left\|x_{n-1}-y_{n-1}\right\|$

Substituting (10) into (9), we have

$\left\|\mathrm{x}_{\mathrm{n}}-\mathrm{y}_{\mathrm{n}}\right\| \leq \alpha_{\mathrm{n}-1} \varphi\left(\left\|\mathrm{z}_{\mathrm{n}-1}-\mathrm{T} \mathrm{z}_{\mathrm{n}-1}\right\|\right)$

$+b \alpha_{n-1} \beta_{n-1} \varphi\left(\left\|x_{n-1}-T x_{n-1}\right\|\right)+$

$\left(1-\alpha_{\mathrm{n}-1}+\mathrm{b} \alpha_{\mathrm{n}-1}\right)\left\|\mathrm{x}_{\mathrm{n}-1}-\mathrm{y}_{\mathrm{n}-1}\right\|+\varepsilon_{\mathrm{n}-1}$

Substituting (11) into (8) yields:

$\left\|y_{n+1}-p\right\|_{\leq} \leq\left\|x_{n+1}-p\right\|+\alpha_{n} \varphi\left(\left\|z_{n}-T z_{n}\right\|\right)+b \alpha_{n} \beta_{n} \varphi$ $\left(\left\|\mathrm{x}_{\mathrm{n}}-\mathrm{T} \mathrm{x}_{\mathrm{n}}\right\|\right)+\varepsilon$

$+\left(1-\alpha_{n}+b \alpha_{n}\right) \varepsilon_{n-1}+\left(1-\alpha_{n}+b \alpha_{n}\right) \alpha_{n-1} \varphi\left(\left\|z_{n-1}-T z_{n-1}\right\|\right)$

$+b\left(1-\alpha_{n}+b \alpha_{n}\right) \alpha_{n-1} \beta_{n-1} \varphi\left(\left\|x_{n-1}-T_{n-1}\right\|\right)$

$+\left(1-\alpha_{n}+b \alpha_{n}\right)\left(1-\alpha_{n-1}+b \alpha_{n-1}\right)\left\|x_{n-1}-y_{n-1}\right\|$

Repeating this process (n-1) more times yields (5).

This completes the proof.

Remark 2: If $\beta_{n}=0$ in Lemma 2, then we obtain an equivalent result for the Mann iteration process.

\section{MAIN RESULTS}

Theorem 1: Let $(\mathrm{E},\|\|$.$) be a normed linear space$ and let $\mathrm{T}: \mathrm{E} \rightarrow \mathrm{E}$ be a selfmap of $\mathrm{E}$ satisfying the contractive definition (4). Suppose $\mathrm{T}$ has a fixed point $\mathrm{p}$ and the sequence $\left\{\mathrm{x}_{\mathrm{n}}\right\}_{n=o}^{\infty}$ is the Ishikawa iteration process satisfying the conditions of Lemma 2. Then, the Ishikawa iteration process is T-stable.

Proof: Suppose $\lim _{n \rightarrow \infty} \mathcal{E}_{n}=0$. Then, we shall show that $\lim _{n \rightarrow \infty} y_{n}=p$, using Lemmas 1 and 2. Let $\mathrm{C}$ be the lower triangular matrix with entries:

$\mathrm{c}_{\mathrm{nj}}=\alpha_{\mathrm{j}} \Pi_{k=j+1 o}^{n}\left(1-\alpha \mathrm{k}^{+} \quad \mathrm{b} \alpha_{\mathrm{k}}\right) . \quad$ Then, $\mathrm{C}$ is multiplicative ${ }^{[1,4]}$. Since $\varphi$ is continuous and $\lim _{n \rightarrow \infty} \| z_{n}$ $\mathrm{Tz}_{\mathrm{n}} \|=0$, then by Lemma 1 , we obtain:

$$
\begin{aligned}
& \lim _{n \rightarrow \infty} \sum_{j=0}^{n} \alpha_{j} \prod_{k=j+1}^{n}\left(1-\alpha_{\mathrm{k}}+\mathrm{b} \alpha_{\mathrm{k}}\right) \varphi \\
& \left(\left\|\mathrm{z}_{\mathrm{j}}-\mathrm{Tz}_{\mathrm{j}}\right\|\right)=0 .
\end{aligned}
$$

Furthermore,

$$
\begin{aligned}
& 0 \leq b \sum_{j=0}^{n} \alpha_{j} \prod_{k=j+1}^{n}\left(1-\alpha_{\mathrm{k}}+\mathrm{b} \alpha_{\mathrm{k}}\right) \varphi\left(\left\|\mathrm{x}_{\mathrm{j}}-\mathrm{Tx}_{\mathrm{j}}\right\|\right) \\
& \leq b \sum_{j=0}^{n} \alpha_{j} \prod_{k=j+1}^{n}\left(1-\alpha_{\mathrm{k}}+\mathrm{b} \alpha_{\mathrm{k}}\right) \varphi\left(\left\|\mathrm{x}_{\mathrm{j}}-\mathrm{Tx}_{\mathrm{j}}\right\|\right) .
\end{aligned}
$$

Since $\varphi$ is continuous and $\left.\lim _{n \rightarrow \infty}\left\|\mathrm{x}_{\mathrm{n}}-\mathrm{Tx}_{\mathrm{n}}\right\|\right)=0$, we have

$\lim _{n \rightarrow \infty} b \sum_{j=0}^{n} \alpha_{j} \prod_{k=j+1}^{n}\left(1-\alpha_{\mathrm{k}}+\mathrm{b} \alpha_{\mathrm{k}}\right) \varphi$

$\left(\left\|\mathrm{x}_{\mathrm{j}}-\mathrm{Tx}_{\mathrm{j}}\right\|\right)=0$.

which implies that :

$\lim _{n \rightarrow \infty} b \sum_{j=0}^{n} \alpha_{j} \beta_{j} \prod_{k=j+1}^{n}$

$\left(1-\alpha_{k}+b \alpha_{k}\right) \varphi\left(\left\|x_{j}-T x_{j}\right\|\right)=0$,

Let $\mathrm{D}$ be the lower triangular matrix with entries $\mathrm{d}_{\mathrm{nj}}=\prod_{k=j+1}^{n}\left(1-\alpha_{\mathrm{k}}+\mathrm{b} \alpha_{\mathrm{k}}\right)$.

Condition (iv) of Lemma 2 implies that $\mathrm{D}$ is multiplicative $\mathrm{e}^{[5]}$ and since $\lim _{n \rightarrow \infty} \varepsilon_{n}=0$, we obtain:

$\lim _{n \rightarrow \infty} \sum_{j=o}^{n} \prod_{k=j+1}^{n}\left(1-\alpha_{\mathrm{k}}+\mathrm{b} \alpha_{\mathrm{k}}\right) \varepsilon_{n}=0$

Moreover, condition (iii) of Lemma 2 implies that $\lim _{n \rightarrow \infty} \prod_{k=o}^{n}\left(1-\alpha_{\mathrm{k}}+\mathrm{b} \alpha_{\mathrm{k}}\right)=0$.

Also, we shall prove that $\lim _{n \rightarrow \infty}\left\|\mathrm{x}_{\mathrm{n}+1}-\mathrm{p}\right\|=0$.

Using (4), triangle inequality and condition (ii) of Lemma 2, we have:

$$
\begin{aligned}
& \left\|\mathrm{x}_{\mathrm{n}+1}-\mathrm{p}\right\|=\left\|\left(1-\alpha_{\mathrm{n}}\right) \mathrm{x}_{\mathrm{n}}+\alpha_{\mathrm{n}} \mathrm{T} \mathrm{z}_{\mathrm{n}}-\mathrm{p}\right\| \\
& =\left\|\left(1-\alpha_{n}\right)\left(x_{n}-p\right)+\alpha_{n}\left(T z_{n}-p\right)\right\| \\
& =\left\|\left(1-\alpha_{n}\right)\left(x_{n}-p\right)+\alpha_{n}\left(T z_{n}-T p\right)\right\| \\
& \leq\left(1-\alpha_{n}\right)\left\|x_{n}-p\right\|+\alpha_{n}\left\|T p-T z_{n}\right\| \\
& \leq\left\|\left(1-\alpha_{n}\right)\right\| x_{n}-p \|+\alpha_{n}[\varphi(\|p-T p\|) \\
& \left.+b\left\|p-z_{n}\right\|\right] \\
& \left.=\left\|\left(1-\alpha_{n}\right)\right\| x_{n}-p\left\|+b \alpha_{n}\right\| 1-\beta_{n}\right) x_{n} \\
& +\beta_{\mathrm{n}} \mathrm{Tx}_{\mathrm{n}}-\mathrm{p} \| \\
& =\left\|\left(1-\alpha_{n}\right)\right\| x_{n}-p \|+b \alpha_{n}\left[\| 1-\beta_{n}\right)\left(x_{n}-p\right) \\
& +\beta_{\mathrm{n}}\left(\mathrm{Tx}_{\mathrm{n}}-\mathrm{p}\right) \\
& \left.\leq\left\|\left(1-\alpha_{n}\right)\right\| x_{n}-p\left\|+b \alpha_{n}\left(1-\beta_{n}\right)\right\| x_{n}-p \|\right) \\
& \left.+\mathrm{b} \alpha_{\mathrm{n}} \beta_{\mathrm{n}}\right)\left\|\mathrm{Tp}-\mathrm{Tx}_{\mathrm{n}}\right\| \\
& \left.\leq\left\|\left(1-\alpha_{\mathrm{n}}\right)\right\| \mathrm{x}_{\mathrm{n}}-\mathrm{p}\left\|+\mathrm{b} \alpha_{\mathrm{n}}\left(1-\beta_{\mathrm{n}}\right)\right\| \mathrm{x}_{\mathrm{n}}-\mathrm{p} \|\right) \\
& +b \alpha_{n} \beta_{n}\left[\varphi(\|p-T p\|)+b\left\|p-x_{n}\right\|\right] \\
& =\left(1-\alpha_{n}+b \alpha_{n}\right)\left\|x_{n}-p\right\|-b \alpha_{n} \beta_{n}(1-b)\left\|x_{n}-p\right\| \\
& \leq\left[1-(1-b) \alpha_{n}\right]\left\|x_{n}-p\right\| \\
& =\exp \left(-(1-b) \alpha_{n}\right)\left\|\mathrm{x}_{\mathrm{n}}-\mathrm{p}\right\| \\
& \leq \exp \left(-(1-b) \alpha_{n}\right) \exp \left(-(1-b) \alpha_{n-1}\right)\left\|x_{n-1}-p\right\| \\
& \leq \exp \left(-(1-b) \alpha_{n}\right) \exp \left(-(1-b) \alpha_{n-1}\right) \ldots \leq \\
& \exp \left(-(1-b) \alpha_{o}\right)\left\|\mathrm{x}_{0}-\mathrm{p}\right\| \\
& =\exp \left(-(1-\mathrm{b}) \sum_{j=0}^{n} \alpha_{j}\right)\left\|\mathrm{x}_{\mathrm{o}}-\mathrm{p}\right\| \rightarrow 0 \text {, } \\
& \text { as } \mathrm{n} \rightarrow \infty \text {. }
\end{aligned}
$$

Hence, inequality (5) yields $\lim _{n \rightarrow \infty} y_{n}=p$.

Conversely, suppose that $\lim _{n \rightarrow \infty} y_{n}=p$. Then,

$\varepsilon_{\mathrm{n}}=\left\|\mathrm{y}_{\mathrm{n}+1}-\left(1-\alpha_{\mathrm{n}}\right) \mathrm{y}_{\mathrm{n}}-\alpha_{\mathrm{n}} \mathrm{Ts}_{\mathrm{n}}\right\|$

$\leq\left\|y_{n+1}-p\right\|+\left\|p-\left(1-\alpha_{n}\right) y_{n}-\alpha_{n} T s_{n}\right\|$

$=\left\|y_{n+1}-p\right\|+\left\|\left(1-\alpha_{n}\right)\left(y_{n}-p\right)+\alpha n\left(T s_{n}-p\right)\right\|$ 


$$
\begin{aligned}
& \left\|\begin{array}{l}
y_{n+1}-p \\
y_{n+1}-p
\end{array}++\left(1-\alpha_{n}\right)\right\| y_{n}-p\left\|+\alpha_{n}\right\| T p-T s_{n} \| \\
& \left.\left.+b \| p-\alpha_{n}\right) \|\right) \\
& +\left\|y_{n}-p\right\|+\alpha_{n}(\varphi(\|p-T p\|) \\
& =\left\|y_{n+1}-p\right\|+\left(1-\alpha_{n}\right)\left\|y_{n}-p\right\|+b \alpha_{n}\left(1-\beta_{n}\right)\left(y_{n}-p\right) \\
& +\beta_{n}\left(T y_{n}-p\right) \| \\
& \left\|y_{n+1}-p\right\|+\left(1-\alpha_{n}\right)\left\|y_{n}-p\right\|+b \alpha_{n}\left(1-\beta_{n}\right)\left(y_{n}-p\right) \\
& +b \alpha_{n} \beta_{n}\left\|T p-T y_{n}\right\| \\
& \left\|y_{n+1}-p\right\|+\left(1-\alpha_{n}+b \alpha_{n}-b \alpha_{n} \beta_{n}\right)\left\|\left(y_{n}-p\right)\right\| \\
& +b \alpha_{n} \beta_{n}\left(\varphi\left(\|p-T p\|+b\left\|p-y_{n}\right\|\right)\right) \\
& =\left\|y_{n+1}-p\right\|+\left(1-\alpha_{n}+b \alpha_{n}-b \alpha_{n} \beta_{n}+b^{2} n \beta_{n}\right) \\
& \left\|\left(y_{n}-p\right)\right\| \rightarrow 0, \text { as } n \rightarrow \infty
\end{aligned}
$$

This completes the proof of the Theorem.

Remark 3: Theorem 1 is a generalization of Theorem 2 of Osilike ${ }^{[1]}$ and Theorem 30 of Rhoades ${ }^{[3]}$. If $\beta_{n}=$ $0, \forall \mathrm{n} \geq 0$ in Theorem 1, we obtain a generalization of Theorem 2 of Rhoades $^{[4]}$ which itself is a generalization of both Theorem 3 of Harder and Hicks $^{[2]}$ and Theorem 2 of Rhoades ${ }^{[5]}$.

By Remark 2, we have the following stability result for the Mann iteration process.

Corollary 1: Let $(\mathrm{E},\|\|$.$) be a normed linear space$ and let $\mathrm{T}: \mathrm{E} \rightarrow \mathrm{E}$ be a selfmap of $\mathrm{E}$ satisfying the contractive definition (4). Suppose $\mathrm{T}$ has a fixed point $\mathrm{p}$ and let be the Mann iteration process satisfying the conditions of Remark 2. then, the Mann iteration process is T-stable.
Proof: The proof follows directly from Theorem 1, by putting $\beta_{\mathrm{n}}=0$.

Remark 4: Corollary 1 is a generalization of Theorem 2 of Rhoades ${ }^{[4]}$, which itself is a generalization of both Theorem 3 of Harder and Hicks ${ }^{[2]}$ and Theorem 2 of Rhoades $^{[5]}$.

\section{REFERENCES}

1. Osilike, M.O., 1995. Stability results for fixed point iteration procedure. J. Nigerian Math. Soc., 14/15: 17-29.

2. Harder, A.M. and T.L. Hicks, 1988. Stability results for fixed point iteration procedures, Math. Japonica 33: 693-706.

3. Rhoades, B.E., 1991. Some fixed point iteration procedures. Intl. J. Math. Math. Sci., 4: 1-16.

4. Rhoades, B.E., 1993. Fixed point theorems and stability results for fixed point iteration procedures II. Indian J. Pure and Applied Math., 24: 691-703 .

5. Rhoades, B.E., 1990. Fixed point theorems and stability results for fixed point iteration procedures I. Indian J. Pure and Applied Math., 21: 1-9.

6. Rhoades, B.E., 1976. Comments on two fixed point iteration methods. J. Math. Anal. Appl., 56: 741-750.

7. Berinde, V., 2002. On the stability of some fixed point procedures. Bul. Stiint. Univ. Baia Mare, Ser.B, Matematica-Informatica, xviii: 7-14. 